\title{
Smart knife: technological advances towards smart cutting tools in meat industry automation
}

\author{
Alex Mason \\ Faculty of Science and Technology, Norwegian University of Life Sciences, Ås, Norway and Research and Development, Animalia AS, \\ Oslo, Norway \\ Dmytro Romanov, L. Eduardo Cordova-Lopez and Steven Ross \\ Faculty of Science and Technology, Norwegian University of Life Sciences, Ås, Norway, and \\ Olga Korostynska \\ Department of Mechanical, Electronics and Chemical Engineering at OsloMet, Oslo, Norway
}

\begin{abstract}
Purpose - Modern meat processing requires automation and robotisation to remain sustainable and adapt to future challenges, including those brought by global infection events. Automation of all or many processes is seen as the way forward, with robots performing various tasks instead of people. Meat cutting is one of these tasks. Smart novel solutions, including smart knives, are required, with the smart knife being able to analyse and predict the meat it cuts. This paper aims to review technologies with the potential to be used as a so-called "smart knife" The criteria for a smart knife are also defined.

Design/methodology/approach - This paper reviews various technologies that can be used, either alone or in combination, for developing a future smart knife for robotic meat cutting, with possibilities for their integration into automatic meat processing. Optical methods, Near Infra-Red spectroscopy, electrical impedance spectroscopy, force sensing and electromagnetic wave-based sensing approaches are assessed against the defined criteria for a smart knife.

Findings - Optical methods are well established for meat quality and composition characterisation but lack speed and robustness for real-time use as part of a cutting tool. Combining these methods with artificial intelligence (Al) could improve the performance. Methods, such as electrical impedance measurements and rapid evaporative ionisation mass spectrometry, are invasive and not suitable in meat processing since they damage the meat. One attractive option is using athermal electromagnetic waves, although no commercially developed solutions exist that are readily adaptable to produce a smart knife with proven functionality, robustness or reliability.

Originality/value - This paper critically reviews and assesses a range of sensing technologies with very specific requirements: to be compatible with robotic assisted cutting in the meat industry. The concept of a smart knife that can benefit from these technologies to provide a real-time "feeling feedback" to the robot is at the centre of the discussion.
\end{abstract}

Keywords Meat industry, Automation, Robotics, Smart knife, Sensing principles, Electromagnetic waves, NIR, Optical methods, Spectroscopy

Paper type Literature review

\section{Introduction}

A butcher uses a combination of both the eye-vision and handfeeling to analyse the material being cut, to differentiate between the layers in the complex medium (e.g. a carcass) and make instant decisions. When using a knife, a professional butcher always "feels" what is being cut: a layer of skin, muscle, fatty tissue or a bone - all will have different effect on the same knife. Based on this "feedback effect" a person knows what to do - to proceed the same way, to change the force, to alter the

The current issue and full text archive of this journal is available on Emerald Insight at: https://www.emerald.com/insight/0260-2288.htm

Sensor Review

42/1 (2022) 155-163

Emerald Publishing Limited [ISSN 0260-2288]

[DOI 10.1108/SR-09-2021-0315] manner of cutting or to stop. But can a robot do that? And if so, how?

This paper critically reviews various sensing principles that can be used to enable real-time "feeling" feedback in automated meat cutting applications. The applicability of

(C) Alex Mason, Dmytro Romanov, L. Eduardo Cordova-Lopez, Steven Ross and Olga Korostynska. Published by Emerald Publishing Limited. This article is published under the Creative Commons Attribution (CC BY 4.0) licence. Anyone may reproduce, distribute, translate and create derivative works of this article (for both commercial and non-commercial purposes), subject to full attribution to the original publication and authors. The full terms of this licence maybe seen at http:// creativecommons.org/licences/by/4.0/legalcode

The work is funded by the European Commission H2020 project RoBUTCHER (Grant Agreement \#871631). https://robutcher.eu/.

Received 17 September 2021

Revised 9 November 2021

Accepted 20 November 2021 
approaches used in other areas is analysed, with assessment made considering the limitations imposed by the meat industry traditions and regulations. It is noted that the authors are primarily engaged in automation research and development associated with abattoirs and therefore this paper often refers to primary processing; for example, removal of primals such as limbs from a carcass. Nevertheless, the ideas and concepts discussed in the paper should have relevance throughout the primary and secondary processing stages of the meat value chain, regardless of species.

The cuts made with a knife during any meat cutting process vary in complexity. It is therefore perceived that smart feedback should be incorporated in future advanced robot systems, so that the robot can adapt to any variation (e.g. biological) presented and provide better yield than if it were to cut blindly based on some simple "rule of thumb". Conversely, the operations with a knife (e.g. limb removal) are considered complex and therefore information regarding the material in immediate proximity to the knife is essential to achieve efficient automation in the meat industry.

Notably, the reality is that the visual part of the cutting process is a preliminary step, to assess the situation. However, feeling is the main "in operation" feedback. Based on the extensive discussions with the professional butchers, vision plays a very small role after an initial decision of how to proceed and is often too slow, or even late, to help because the butcher cannot see the tip of the knife when it is buried within the meat mid-cut. Aside from this, purely optical methods with traditional camera systems used for the image analysis are not adequate for the task in hand. Often camera views are easily obscured by robots, in-process tools, or a camera lens may be contaminated with blood and distort the image, resulting in poor quality data. Furthermore, most general-purpose cameras (e.g. Intel Realsense) cannot focus on the work object once a certain minimum distance threshold has been passed, ruling out up-close visual monitoring of the knife. Therefore, traditional optical imaging approaches are not the main focus of this paper, as the meat industry cannot rely solely on these and new advancements are needed for the smart knife of the future.

The two challenges that so far hinder the process in automated meat cutting are the gathering of reliable information and the correct positioning of the cutting tool and these tasks are by no means trivial. A wide range of techniques, from low frequency, high frequency electrical impedance measurement, microwaves, NMR, IR and UV light, to X-rays, involves a wide range of physical interactions between the sensing method and the sample. However, not all these approaches are suitable for real-time haptics-cut feedback during actual meat cutting. Only those that offer an instantaneous response and a non-destructive interaction mechanism, are considered in this paper as potentially feasible options to advance and customise meat industry automation. Moreover, these methods should not affect the meat itself (Egelandsdal et al., 2019) in a way that could change product properties (Muradov et al., 2020), or even render some parts of it unsuitable for consumption.

To decide which technologies are potentially feasible for realisation in a "smart knife" concept, certain criteria should be met, such as those listed in Table 1. Notably, the focus is specifically on a knife that is attached to a robotic arm and has a built-in sensing mechanism that provides the robotic system with the real-time information on what material (meat, fat, bone and so forth) is in direct contact or immediate proximity to that knife, so that the cutting trajectory can be adjusted and cutting activities coordinated. Figure 1(a) illustrates a custommade knife (Uddeholm AB, Sweden) attached to a Universal Robot (UR10), while Figure 1(b) depicts this knife during the action of meat cutting.

\section{Electrical impedance measurements}

Electrical impedance measurement as an indication of meat properties has been considered previously, an example of which is shown in Figure 2. The structural organisation and composition of meat makes it a highly anisotropic dielectric material, i.e. electrical impedance varies according to whether the current runs parallel or perpendicular to muscle fibres (Damez and Clerjon, 2013).

As fat is an electrical insulator, it influences the electrical impedance of the tissue. Attempts to use electrical properties to estimate fat content in animal carcasses and meat are known (Slanger and Marchello, 1994). Notably, the latency and invasiveness of measurements render this approach impractical for high-value automated meat cutting, as measuring electrical

Table 1 Criteria for sensing approaches for smart knife implementation

\begin{tabular}{|c|c|}
\hline Criterium & Description \\
\hline No damage & Technology used to assist a smart knife to sense what is being cut should not damage or change the meat sample in any way \\
\hline Speed & The sensing method should be sufficiently fast to provide real-time data regarding the material and/or cutting characteristics \\
\hline Size & $\begin{array}{l}\text { The sensor size should be compatible with that of the knife and integrated within the blade, attached to the robotic arm, without } \\
\text { impeding either the knife blade or the robot }\end{array}$ \\
\hline Material & $\begin{array}{l}\text { The blade should be the optimal compromise of hardness, thickness, elasticity and form to be able to perform all necessary cuts. The } \\
\text { material used to fabricate the smart knife should be suitable to comply with strict food hygiene regulations. Moreover, this material } \\
\text { should be cleanable using approved procedures to eliminate the potential for cross-contamination between the meat samples from } \\
\text { various carcasses, from human operators or robotic surfaces }\end{array}$ \\
\hline Cost & $\begin{array}{l}\text { The sensing technology used for a smart knife solution should be cost-effective, so as not to render the intended benefits of } \\
\text { automated cutting invalid, specifically in meat industry, where the profit margins are low }\end{array}$ \\
\hline Depth & $\begin{array}{l}\text { Meat is a non-uniform material. Therefore, the technology should be able to accurately sense particularly characteristics of a cut, } \\
\text { namely that the knife is in contact with the meat and its cutting depth. This will enable in-process adjustment of planned robot paths }\end{array}$ \\
\hline
\end{tabular}


Figure 1 Custom-made knife attached to Universal Robot (UR10) (a) and cutting a sample of meat (b)

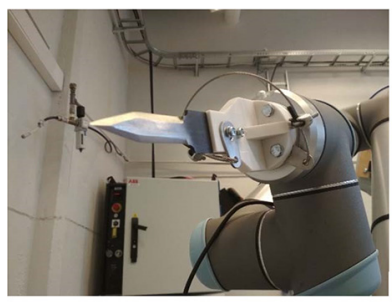

(a)

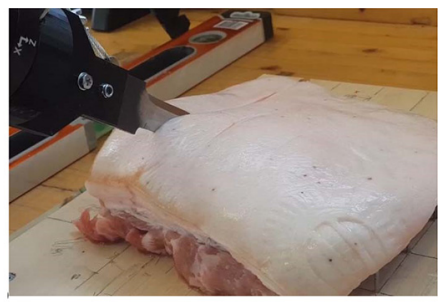

(b)
Figure 2 Electrical impedance measurement concept: muscle fibres in meat can be represented as a resistor and its response to the current depends on orientation and properties of these fibres

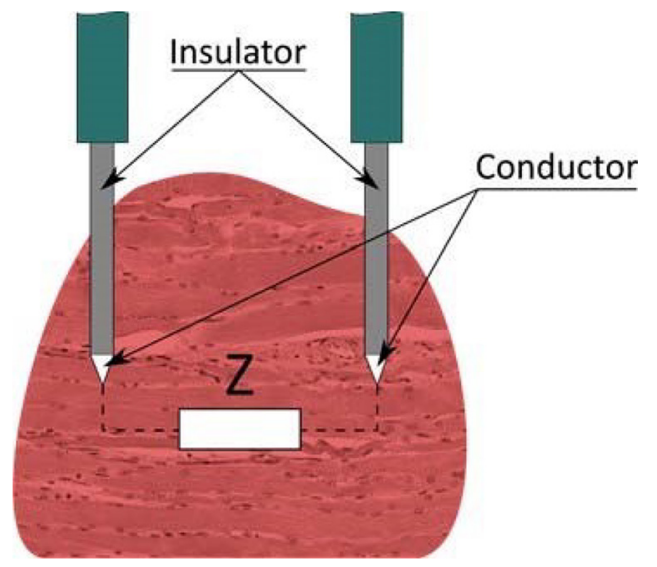

impedance would involve introducing physical damage to the meat, as it requires the direct contact of probes that pierce deep within the tissue to allow current to pass. Moreover, it was reported that there is no relationship between electrical conductivity and shear force values, promoting the argument that the amount of connective tissue inducing meat toughness cannot be assessed by a straightforward electrical measurement (Byrne et al., 2000).

While capacitive or conductivity sensing are often used to detect proximity or touch, these methods may be challenging to apply because it is difficult to ensure reliable grounding conditions for food applications.

\section{Force sensing}

A knife with embedded force sensors is another viable approach to help determine the actual type of material being cut. In medicine, these sensorised tools are already used for performing robot-assisted surgeries. The novel technology for two degree-of-freedom (DOF) lateral force sensing reported by Shahzada et al. (2016) uses a novel layout of four fibre Bragg grating (FBG) sensors attached to the shaft of a da Vinci ${ }^{\circledR}$ surgical instrument.

In the meat industry, the application of force sensing assumes that fat, meat and cartilage all require different force to cut through and measuring this force will in turn inform on the type of the tissue in contact with the knife. A graphical concept of this approach is given in Figure 3.

The idea was mainly for the "assistive kitchen" application, still operated by a human (Beetz et al., 2008), but robotassisted automatic cutting is also an attractive option in this scenario. There are numerous developments in force sensing for robotic arm application, with some recent examples being (Bimbo et al., 2017; Bouteraa and Ben Abdallah, 2017; Jawale et al., 2019; Tae Mun et al., 2016). However, the force sensing approach has no potential for prediction or foresight of the material to be cut. The reason for this is due to material type variation along the blade length being difficult to distinguish and therefore separate. Other possible issues when using smart knife based on built-in force sensing approach for automatic fast-speed cutting are with the response time of the system plus the cost of the sensors. Indicative prices for some commercially available force sensors suitable for this task are given in Table 2. In addition, variations in carcass temperature, density and other important traits (e.g. water holding capacity) of meat (or fat) between different pigs are factors affecting automation. As a result, each time the sensors are used a calibration is required and that could hinder the automation process. Force measurements could also be misinterpreted, as the force applied at the tip of a knife is not the same as along the rest of the blade. When attached to a robotic arm with multiple degrees of freedom (DoF), the orientation of the cut could potentially affect the interpretation of measurements (Chen et al., 2021). Thus, no absolute force value can be used for a certain type of meat tissue and for each type the sensor system must be recalibrated, precluding efficient automation.

Combination with intelligent data processing and specifically trained neural networks can significantly increase the accuracy and reliability of force measurements for automated meat cutting. The two assistive strategies, namely, a force amplification strategy and an intent prediction strategy using an unrolled Recurrent Neural Network were illustrated in Maithani et al. (2021). They enable a KUKA LWR robot to provide assistive forces to a professional butcher while simultaneously allowing motion of the knife in all degrees of freedom. This prototype was specifically designed for a collaborative robot in meat cutting tasks to reduce the likelihood of musculoskeletal disorders in the wrist of human operators working in the meat industry. It is yet to be seen if the same approach can be further developed to a level suitable for a smart knife in completely robotised meat processing.

\section{Optical methods}

\subsection{Infrared spectroscopy}

Short wavelengths in the NIR (700 to $1100 \mathrm{~nm}$ ) range can penetrate deeply into the skin, offering a potential spectral window for the analysis of animal and human tissues (Roberts et al., 2017). Various optical detection methods are broadly used to assess tissue type - in these cases the specific goal is to determine the quality of the meat. Infrared (IR) spectroscopy is based on the principle that the chemical bonds in organic molecules absorb or emit infrared light when their vibrational state changes. Large changes in vibrational state are observed in the near IR part of the spectrum (NIR) and sometimes up to the visible region, while primary vibrations are produced in the 
Figure 3 Force sensing element is embedded into a knife to assess the cutting process

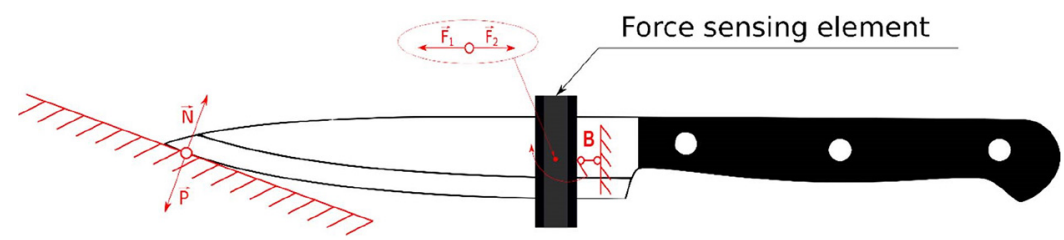

Notes: A range of forces experienced by the knife blade(B) should be measured by the force sensing element: normal $\left(N^{\rightarrow}\right)$, perpendicular $\left(P^{\rightarrow}\right)$ and directional $\left(F^{\rightarrow} 1\right.$ and $F^{2} 2$ )

Table 2 Indicative prices for some commercially available force sensors for fitting on robotic arm

\begin{tabular}{llr}
\hline Company & Sensor & Cost US\$ \\
\hline ABB & Force Sensor Medium & $\sim 10830$ \\
Hypersen Technologies & HPS-FT120 & $\sim 3975$ \\
ATI and Schunk & FTD-Delta SI-660-60 & $\sim 10215$ \\
\hline
\end{tabular}

mid IR region. A major challenge in applying IR spectroscopy to animal production is sample presentation, as transmission is inappropriate for opaque solids. Several IR spectroscopy techniques exist. The main differences are the bandwidth and the optically measured parameters (transmission, reflection, diffusion, scattering, etc.).

Application of visible or near-infrared (Vis-NIR) spectroscopy for meat analysis was, for example, reviewed in Prieto et al. (2009), where the opinion was expressed that over the past three decades, near infrared reflectance spectroscopy (e.g. $700-1100 \mathrm{~nm}$ ) has been proven to be one of the most efficient and advanced tools for the estimation of quality attributes in meat and meat products. There is substantial progress in using NIR techniques to predict chemical composition (crude protein, intramuscular fat, moisture/dry matter, ash, gross energy, myoglobin and collagen), technological parameters $(\mathrm{pH}$ value, colour values, water holding capacity, Warner-Bratzler and slice shear force) and sensory attributes (colour, shape, marbling, odour, flavour, juiciness, tenderness or firmness). The main industrial use of NIR today is for categorising meat into quality classes (Berri et al., 2019; Teixeira et al., 2019). The right of consumers to get exactly the quality they pay for and not an inferior quality meat, drives research in this area.

Recent applications of NIR spectroscopy to predict traits (e.g. protein, fat, fatty acids and shear force) associated with meat quality in both live animals and carcass samples were examined (Chapman et al., 2020). The conclusion was made that, presently, there is a lack of knowledge regarding the capability of rapid and non-invasive methods based on NIR spectroscopy to assess and measure traits associated with meat quality (Prieto et al., 2018). Changes in meat in the time immediately post slaughter are also known to affect the accuracy of the NIRS measurements (Alvarenga et al., 2020).

There is a reduction in accuracy of the NIRS equipment over time, which is a concern for industry needing reliable and consistent standards (Hitchman et al., 2020). However, integrating machine learning techniques could improve the meat quality prediction accuracy. Thus, according to Parastar et al. (2020), handheld spectroscopy was used for monitoring authenticity of packaged chicken fillets and single scans could provide more than $95 \%$ classification accuracy with ensemble learning.

Feasibility assessment of using a commercially available portable pen-sized NIR spectrophotometer and custom made NIR probe with variable distance between light sources specifically for meat type determination was recently reported (Mathew et al., 2021). Attempts to use the commercial instrument to reliably differentiate between various tissues was unsatisfactory and the response accuracy was below the level acceptable for the robotics application in automated meat processing at industrial scale. However, an approach where the distance between the light source and detector can be modified to achieve specific depth of signal penetration seems feasible and needs further development.

Contamination of optical based systems can result in detrimental outputs. The knife blade could be contaminated with blood, muscle, protein and also fat, as it is quite common that some biofilm-like layer forms on the knife after a relatively short period (Giaouris et al., 2014; Keskinen et al., 2008; Wang, 2018). Even immersing a knife blade into hot water $\left(\geq 82^{\circ} \mathrm{C}\right.$ for $\left.5 \mathrm{~s}\right)$ practice does not eliminate microbial contamination of carcasses completely (Durmuşoğlu et al., 2020). Thus, a purely optical solution is not seen as suitable for efficient robotics meat cutting. A combination with other methods may improve this situation, with integration of selflearning AI algorithms being an attractive development (Zhenjie et al., 2019; Swain et al., 2021).

\subsection{Metal embedded optics}

An attempt to develop a smart knife with embedded fibre optics in its blade was an interesting idea (Zoran et al., 2015), the concept is depicted in Figure 4. In this novel technique, the optical fibre embedded blade is used to sense the presence of the objects to be cut. Between the edge of the blade and a knife handle a skin colour sensor operating at the visible-light range of the electromagnetic spectrum was built-in. It aimed to prevent the most common injuries sustained in the kitchen by activating a simple finger-protection mechanism in the form of a retracting blade. This concept, despite being called "a smart knife", so far has not translated into real-world use for a variety of reasons, including complexity and cost of the proposed system. It works by sensing difference in colour between the person's skin and the environment. Attaching cameras to the knife while maintaining a desirable field of view may significantly impact its form factor, therefore it proposed use of an imaging sensor embedded inside the metal of the blade itself. 
Figure 4 Concept of the knife with fibre optics embedded approach

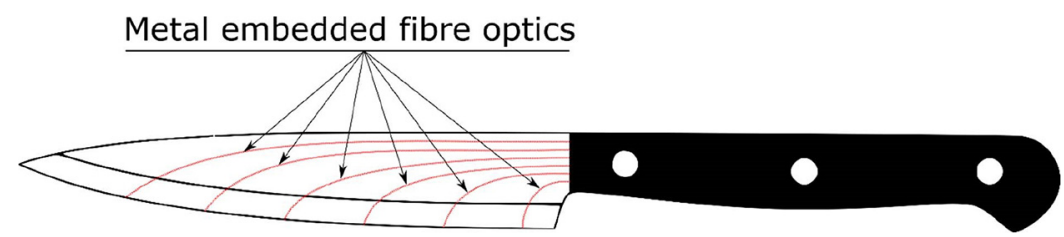

Plastruct FOP-30 Fibre Optics (PMMA acrylic fibres with $0.76 \mathrm{~mm}$ diameter) were chosen over glass-based fibres for their flexibility, durability, multi-modal light transference, thickness and considerably lower price (Zoran et al., 2015). The optic blade included trenches dug into the metal, which guide the fibre optics inside the surface and contained a retracting mechanism. One fibre carried illumination, while the other collected light reflected from objects near the blade.

The authors voiced their suspicions that uncooked pork or chicken meat will prove hard to distinguish from skin colour without a higher-order imaging modality that includes a broader spectrum or improved sensitivity. The system would benefit from an embedded microcontroller with stronger computational power to perform some of the calculations in real time.

Notably, if the fibre optics colour sensor capabilities are improved and it can differentiate between various shades of colours in a meat sample, this approach could be feasible addition in automated meat cutting environment, even as part of a robotic tool to assist in "robotic decision making".

The main purpose of this knife was to minimise cuttingrelated injuries in a traditional human operated kitchen. This is not an issue for the new range of completely robotic kitchens Moley, revealed at the 2021 digital Consumer Electronics Show, developed in collaboration with world-leading German robotic company Schunk. In the Moley kitchen concept it is robots not humans that operate the cutting utensils. However, at the time of its inception customers could not accept the idea of robots wielding knives and so the system adopted a food processor like, closed knife solution. However, new "smart knives" can alleviate fears and stereotypes while, advancing new generations of both meat processing and cooking robots.

\subsection{Polarisation sensitive optical coherence tomography} The fact that the attenuation coefficient in fat is $\approx 9$ times greater than in muscle was explored to employ Polarisation Sensitive Optical Coherence Tomography (PS-OCT) (Thampi et al., 2019) as a tool for assessment of intramuscular fat (IMF) and tenderness of meat samples. PS-OCT is a new fast, noncontact and non-destructive technique with a few micrometres resolution up to a few $\mathrm{mm}$ deep in tissues such as opaque as meat. Also, the phase image relates the changes in the polarisation of light to the birefringence of muscle, which varies with tenderness and can be analysed to predict the tenderness of intact meat samples. The method is made very efficient by extracting relevant information from the three-dimensional high-resolution images generated by OCT using principal component analysis (PCA) (Thampi et al., 2020). The principal components are then used as regressors into a support vector regression (SVR) prediction model. The SVR model was found to predict IMF content stably and accurately, with an $R^{2}$ value of 0.94 . This approach can be seen as interesting for automated, contact-less, non-destructive, real-time classification of the quality of meat samples, but can hardly be used as part of a knife that makes direct contact and cuts the meat and the information on the type of the material being cut is needed immediately, or even better - before the cut is made.

\section{Other spectroscopic measuring options}

\subsection{Rapid evaporative ionisation mass spectrometry}

Another approach of an intelligent knife that can distinguish tissue types is based on rapid evaporative ionisation mass spectrometry (REIMS) and was successfully demonstrated for surgical application, to distinguish normal, borderline and malignant tissues (Phelps et al., 2018). For meat REIMS was used to determine its origin (species, geographical provenance and muscle), optimising meat processing (including ageing type, boar taint detection), predicting meat tenderness, detecting residues in meat such as growth promoting hormones and potentially determining meat safety through identifying metabolites caused by bacteria (Ross et al., 2020). REIMS measures detailed metabolite fingerprints of meat within a few seconds; it is a rapid method and has considerable potential for many on-line applications in the meat industry, as it can perform multiple tests simultaneously from the same sample (Allen, 2021). However, this technique involves heating the tissue to produce aerosol, which is then analysed and heating the meat to such a degree during the automated cutting process is not acceptable as it would destroy or reduce the end-product quality. Thus, this approach is not suitable for a smart knife integration.

\subsection{Thermal detection}

Thermal detection in automated meat processing can only be considered as additional safety feature to detect possible presence of human fingers near the cutting operation (Xiong et al., 2017), but not for distinguishing what is being cut by a knife, as naturally the temperature of the adjacent layers of meat will be similar. Furthermore, one layer (e.g. skin) will obscure the view of thermal imaging sensors from underlying layers (e.g. muscle, bone) (Usamentiaga et al., 2014). A further challenge is the variability in temperature of meat experienced during primary and secondary processing.

\section{Electromagnetic wave-based sensing}

Microwave technology is very safe, posing no threat to operators (Allen, 2021). This technology takes advantage of the differing dielectric properties $\left(\varepsilon^{*}=\varepsilon^{\prime}\right.$-j $\sigma, \varepsilon^{\prime}=$ permittivity and $\sigma=$ conductivity) of biological tissues, where an antenna transmits pulses into the tissues, resulting in a frequency- 
dependent diversion and scattering at the interface between differing tissues (Marimuthu et al., 2021; Mason et al., 2018).

The use of electromagnetic waves in various frequency ranges has been considered for quantifying and predicting the quality of meat products (Damez and Clerjon, 2013; Muradov et al., 2014; Bjarnadottir et al., 2015; Abdullah et al., 2014). The main meat quality traits that can be assessed using electromagnetic waves are sensory characteristics, chemical composition, physicochemical properties, health-protecting properties, nutritional characteristics and safety. Work by Marimuthu et al. (2020) documents efforts to evaluate the ability of portable ultrawide band microwave coupled with a Vivaldi patch antenna to predict fat and tissue depth in lamb carcases, with the same authors reporting the efficiency of this approach for beef (Marimuthu et al., 2021).

There are several values that can be used to describe the dielectric properties of a material, but the most common is its permittivity. In human tissue, it is reported to be ca. 11.3 (average fat), 12.5 (cortical bone), 20.6 (cancellous bone) and 54.8 (muscle) (Hasgall et al., 2018). These values are for materials at room temperature and at the frequency $1 \mathrm{GHz}-$ changing temperature or frequency will result in adjustment of these permittivity values - this is often a source of variation when considering reported values from different authors.

Thus, properties of various pork meat tissues, including fresh and frozen samples, were tested in $0.2-20 \mathrm{GHz}$ frequency range, with the focus on the effect of temperature on dielectric constant (Ngadi et al., 2015). This work has confirmed that dielectric properties could be useful in meat quality and type classification, although meat chilling and heating was used to

Figure 5 Concept of testing the quality of meat with a planar EM sensor with interdigitated electrodes layout

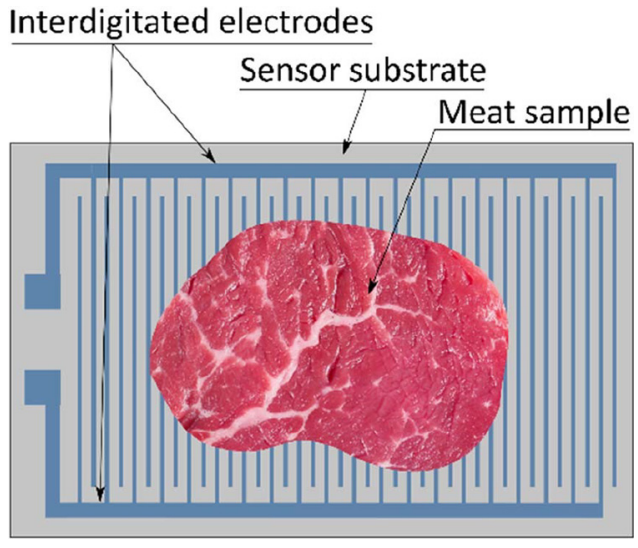

manipulate the meat properties. However, four classes of meat were reliably distinguished based on their dielectric constant and dielectric loss values, depending on the temperature, which was increased up to $85^{\circ} \mathrm{C}$.

The design flexibility offered by microwave sensing to suit a range of applications is another reason why this sensing approach may be considered by the meat industry for assistance in automated cutting to construct an intelligent cutting tool. A notable attempt to use planar electromagnetic sensors for nondestructive testing of meat was reported in Mukhopadhyay et al. (2006). Specifically, a range of meander and mesh type prototype sensors was tested to quantify fat content in meat samples. Both the meander and mesh type sensors consist of two coils: one used for excitation to generate electromagnetic field and another for sensing. The high frequency alternating supply is provided to the exciting coil and the voltage across the sensing coil is measured. The transfer impedance (the ratio of the sensing voltage to the exciting current) is used as the characterisation parameter for the meander and mesh type sensors. In the case of interdigitated sensor, conceptually depicted in Figure 5, the excitation voltage and the displacement current through the sensor were measured. The electrical impedance (the ratio of the applied voltage to the current) was used as the characterisation parameter for the interdigitated sensor.

The results indicated that the response of the sensors for the prediction of fat content in pork belly in a non-invasive way was good at low frequencies, suggesting that it should be possible to develop a low cost sensing system based on a Cygnal microcontroller C8051F020, for example (Mukhopadhyay et al., 2006).

Experimental results with various pork samples, such as skin, fat, muscle and mixed tissue, were generally supportive of the potential of microwave spectroscopy for meat quality analysis. However, the design of the sensors was not further optimised to ensure adequate sensitivity for the tool to be able to characterise tissue types both in real time and with a high degree of certainty. While the proposed planar sensors in concept could be viable for food quality testing, they lack specificity and speed for high-volume robotic meat cutting, as their response relies on the interaction in the contact area between the sensor plane and the meat sample, along with many other variables.

However, the principle of using specific interaction of athermal electromagnetic waves with meat to assess its type is a feasible direction to develop smart knifes with built-in sensing mechanism for real-time analysis and prediction of tissue types in automated cutting applications, for integration with robotic industrial solutions.

Table 3 Benchmarking various sensing technologies with previously set smart knife criteria

\begin{tabular}{|c|c|c|c|c|c|c|}
\hline Sensor technology & No damage & Speed & Size & Material & Cost & $\overline{\text { Depth }}$ \\
\hline Electrical impedance measurements & & & $\sqrt{ }$ & $\sqrt{ }$ & $\sqrt{ }$ & $\sqrt{ }$ \\
\hline Force sensing & $\sqrt{ }$ & & $\sqrt{ }$ & $\sqrt{ }$ & & \\
\hline Optical methods & $\sqrt{ }$ & $\sqrt{ }$ & & $\sqrt{ }$ & & $\sqrt{ }$ \\
\hline Thermal methods & & & & & $\sqrt{ }$ & \\
\hline Electromagnetic spectroscopy & $\sqrt{ }$ & $\sqrt{ }$ & $\sqrt{ }$ & $\sqrt{ }$ & $\sqrt{ }$ & $\sqrt{ }$ \\
\hline
\end{tabular}




\section{Critical comparison of technologies for smart knife}

Meat processing is an industry that requires novel automated solutions to remain sustainable and to mitigate harsh working conditions, shortage of skilled labour and not least, minimise impact of the recent pandemic. The latter has caused closure of many meat factories for hygiene and infection prevention reasons (Rose et al., 2021). Automation of all or many processes is seen as the way forward, with robots performing various tasks instead of people (De Medeiros Esper et al., 2021; Alvseike et al., 2020). Meat cutting is one of these tasks and although many advancements have been made in mechanical approaches to this, the smart cutting tool, or smart knife, is still not a commercially available option.

Each technology reviewed in previous sections has a potential or is already used in the food industry for quality analysis of meat products. However, for meat automation to enable a smart knife solution, only two technologies can be considered for further development, as summarized in Table 3. Specifically, optical and electromagnetic spectroscopies meet all or most of the criteria for smart knife integration, as defined in Table 1.

\section{Conclusion}

Meat processing is an industry that requires novel automated solutions to remain sustainable and to mitigate harsh working conditions, shortage of skilled labour and not least, minimise the impact of recent pandemic, which caused closure of many meat factories for hygiene and infection prevention reasons. Automation of all or many processes is seen as a way forward, with robots performing various tasks alongside, or in the place of human labour. Meat cutting is one of these tasks and although many advancements have been made in mechanical approaches to this, the smart cutting tool or smart knife, is still not a commercially available option. This paper reviews various technologies that can be used for developing the future smart knife for robotic meat cutting. Along with NIR spectroscopy, electromagnetic wave-based sensing is a feasible option that should be developed further to a commercial level. These two approaches could be combined into an advanced multi-sensor system to enable the smart knife, which is very much needed by the robotic solutions in meat cutting. The addition of Artificial Intelligence to assist in decision making and data analysis is an attractive development for the smart knife, to further combine with EM or NIR spectroscopy.

\section{References}

Abdullah, B.M., Cullen, J.D., Korostynska, O., Mason, A. and Al-Shamma'a, A.I. (2014), “Assessing water-holding capacity (WHC) of meat using microwave spectroscopy", in Mason, A., Mukhopadhyay, S.C., Jayasundera, K.P. and Bhattacharyya, N. (Eds), Sensing Technology: Current Status and Future Trends I, Springer International Publishing, Cham, pp. 117-140.

Allen, P. (2021), "Recent developments in the objective measurement of carcass and meat quality for industrial application", Meat Science, Vol. 181, p. 108601.
Alvarenga, T.I.R.C., Hopkins, D.L., Morris, S., Mcgilchrist, P. and Fowler, S.M. (2020), "Intramuscular fat prediction of the semimembranosus muscle in hot lamb carcases using NIR", Meat Science, Vol. 181 No. 108404, pp. 1-7.

Alvseike, O., Prieto, M., Bjørnstad, P.H. and Mason, A. (2020), "Intact gastro-intestinal tract removal from pig carcasses in a novel meat factory cell approach", Acta Veterinaria Scandinavica, Vol. 62 No. 1, pp. 1-4.

Beetz, M., Stulp, F., Radig, B., Bandouch, J., Blodow, N., Dolha, M., Fedrizzi, A., Jain, D., Klank, U., Kresse, I., Maldonado, A., Marton, Z., Mosenlechner, L., Ruiz, F., Rusu, R.B. and Tenorth, M. (2008), "The assistive kitchena demonstration scenario for cognitive technical systems", RO-MAN 2008 - The 17th IEEE International Symposium on Robot and Human Interactive Communication, Vol. 2008, pp. 1-8.

Berri, C., Picard, B., Lebret, B. and ueza, D., Lefèvre, F., Le Bihan-Duval, E., Beauclercq, S., Chartrin, P., Vautier, A., Legrand, I. and Hocquette, J.-F. (2019), "Predicting the quality of meat: myth or reality?", Foods, Vol. 8 No. 10, p. 436.

Bimbo, J., Pacchierotti, C., Aggravi, M., Tsagarakis, N. and Prattichizzo, D. (2017), "Teleoperation in cluttered environments using wearable haptic feedback", 2017 IEEE/ RSf International Conference on Intelligent Robots and Systems (IROS), Piscataway, NF, IEEE, pp. 3401-3408.

Bjarnadottir, S.G., Lunde, K., Alvseike, O., Mason, A. and AlShamma'a, A.I. (2015), "Assessing quality parameters in dry-cured ham using microwave spectroscopy", Meat Science, Vol. 108, pp. 109-114.

Bouteraa, Y. and Ben Abdallah, I. (2017), "A gesture-based telemanipulation control for a robotic arm with biofeedbackbased grasp", Industrial Robot: An International fournal, Vol. 44 No. 5, pp. 575-587.

Byrne, C.E., Troy, D.J. and Buckley, D.J. (2000), "Postmortem changes in muscle electrical properties of bovine $M$. longissimus dorsi and their relationship to meat quality attributes and $\mathrm{pH}$ fall", Meat Science, Vol. 54 No. 1, pp. 23-34.

Chapman, J., Elbourne, A., Truong, V.K. and Cozzolino, D. (2020), "Shining light into meat - a review on the recent advances in in vivo and carcass applications of near infrared spectroscopy", International fournal of Food Science $\mathbb{G}$ Technology, Vol. 55 No. 3, pp. 935-941.

Chen, L., Sun, H., Zhao, W. and Yu, T. (2021), "AI based gravity compensation algorithm and simulation of load end of robotic arm wrist force", Mathematical Problems in Engineering, Vol. 2021, pp. 1-11.

Damez, J.L. and Clerjon, S. (2013), "Quantifying and predicting meat and meat products quality attributes using electromagnetic waves: an overview", Meat Science, Vol. 95 No. 4, pp. 879-896.

De Medeiros Esper, I., From, P.J. and Mason, A. (2021), "Robotisation and intelligent systems in abattoirs", Trends in Food Science E Technology, Vol. 108, pp. 214-222.

Durmuşoğlu, H., İncili, G.K., Demir, P. and İlhak, O.İ. (2020), "Effects of workers' hand washing and knife disinfection practices on microbiological quality of small animal carcasses in slaughterhouse environment", fournal of Food Processing and Preservation, Vol. 44 No. 12, pp. 1-7. 
Egelandsdal, B., Abie, S.M., Bjarnadottir, S., Zhu, H., Kolstad, H., Bjerke, F., Martinsen, Ø.G., Mason, A. and Münch, D. (2019), "Detectability of the degree of freeze damage in meat depends on analytic-tool selection", Meat Science, Vol. 152, pp. 8-19.

Giaouris, E., Heir, E., Hébraud, M., Chorianopoulos, N., Langsrud, S., Møretrø, T., Habimana, O., Desvaux, M., Renier, S. and Nychas, G.-J. (2014), "Attachment and biofilm formation by foodborne bacteria in meat processing environments: causes, implications, role of bacterial interactions and control by alternative novel methods", Meat Science, Vol. 97 No. 3, pp. 298-309.

Hasgall, P. Di Gennaro, F. Baumgartner, C. Neufeld, E. Lloyd, B. Gosselin, M. Payne, D. Klingenböck, A. and Kuster, N. (2018), “IT'IS database for thermal and electromagnetic parameters of biological tissues", itis.swiss/ database.

Hitchman, S., Johnson, P., Bain, W., Craigie, C.R. and Reis, M.M. (2020), "Short communication: long term performance of near infrared spectroscopy to predict intramuscular fat content in New Zealand lamb", Meat Science, Vol. 181, p. 108376.

Jawale, H.P., Jaiswal, A. and Bhasme, K.N. (2019), "Design and analysis of three-axis cantilever type force sensor", World Fournal of Engineering, Vol. 16 No. 4, pp. 497-508.

Keskinen, L.A., Todd, E.C. and Ryser, E.T. (2008), “Transfer of surface-dried listeria monocytogenes from stainless steel knife blades to roast Turkey breast", fournal of Food Protection, Vol. 71 No. 1, pp. 176-181.

Maithani, H., Corrales Ramon, J.A., Lequievre, L., Mezouar, Y. and Alric, M. (2021), "Exoscarne: assistive strategies for an industrial meat cutting system based on physical humanrobot interaction", Applied Sciences, Vol. 11Is. No. 9, p. 3907.

Marimuthu, J., Loudon, K.M.W. and Gardner, G.E. (2020), "Prediction of lamb carcase C-site fat depth and GR tissue depth using a non-invasive portable microwave system", Meat Science, Vol. 181, p. 108398.

Marimuthu, J., Loudon, K.M.W. and Gardner, G.E. (2021), "Ultrawide band microwave system as a non-invasive technology to predict beef carcase fat depth", Meat Science, Vol. 179, p. 108455.

Mason, A., Korostynska, O., Louis, J., Cordova-Lopez, L.E., Abdullah, B., Greene, J., Connell, R. and Hopkins, J. (2018), "Noninvasive in-situ measurement of blood lactate using microwave sensors", IEEE Transactions on Biomedical Engineering, Vol. 65 No. 3, pp. 698-705.

Mathew, A., Hassan, H.W., Mirtaheri, P. and Korostynska, O. (2021), "Feasibility of using NIR spectroscopy in automated meat cutting", in Haidegger, T., Mason, A., Ross, S. and Takács, K. (Eds), Workshop "Challenges in Automated Food Processing, European Robotics Forum (ERF 2021), Obuda University, Budapest, pp. 10-12.

Mukhopadhyay, S.C., Gooneratne, C.P. and Gupta, G.S. (2006), "Non-invasive testing of quality of pork meat: novel planar electromagnetic sensors based approach", 2006 IEEE Instrumentation and Measurement Technology Conference Proceedings, pp. 1947-1952.

Muradov, M., Cullen, J.D., Abdullah, B., Ateeq, M., Mason, A., Shaw, A. and Al-Shamma'a, A.I. (2014), "Real-time monitoring of meat drying process using microwave spectroscopy", 8th International Conference on Sensing Technology, IEEE Computer Society, ICST 2014, Liverpool, pp. 407-411.

Muradov, M., Cullen, J.D., Abdullah, B., Atteq, M., Mason, A., Shaw, A. and Al-Shamma'a, A.I. (2020), "Real-time monitoring of meat drying process using microwave spectroscopy", International fournal on Smart Sensing and Intelligent Systems, Vol. 7 No. 5, pp. 1-5.

Ngadi, M., Dev, S.R.S., Raghavan, V.G.S. and Kazemi, S. (2015), "Dielectric properties of pork muscle", International Fournal of Food Properties, Vol. 18 No. 1, pp. 12-20.

Parastar, H., Van Kollenburg, G., Weesepoel, Y., Van Den Doel, A., Buydens, L. and Jansen, J. (2020), "Integration of handheld NIR and machine learning to 'measure \& monitor' chicken meat authenticity", Food Control, Vol. 112, p. 107149.

Phelps, D.L., Balog, J., Gildea, L.F., Bodai, Z., Savage, A., ElBahrawy, M.A., Speller, A.V., Rosini, F., Kudo, H., Mckenzie, J.S., Brown, R., Takáts, Z. and GhaemMaghami, S. (2018), "The surgical intelligent knife distinguishes normal, borderline and malignant gynaecological tissues using rapid evaporative ionisation mass spectrometry (REIMS)", British fournal of Cancer, Vol. 118 No. 10, pp. 1349-1358.

Prieto, N., Roehe, R., Lavín, P., Batten, G. and Andrés, S. (2009), "Application of near infrared reflectance spectroscopy to predict meat and meat products quality: a review", Meat Science, Vol. 83 No. 2, pp. 175-186.

Prieto, N., Dugan, M.E.R., Juárez, M., López-Campos, Ó., Zijlstra, R.T., Aalhus, J.L. and Plaizier, J. (2018), "Using portable near-infrared spectroscopy to predict pig subcutaneous fat composition and iodine value", Canadian Fournal of Animal Science, Vol. 98 No. 2, pp. 221-229.

Roberts, J.J., Motin, J.C., Swain, D. and Cozzolino, D. (2017), "A feasibility study on the potential use of near infrared reflectance spectroscopy to analyze meat in live animals: discrimination of muscles", fournal of Spectroscopy, Vol. 2017, p. 3948708.

Rose, D.C., Lyon, J., De Boon, A., Hanheide, M. and Pearson, S. (2021), "Responsible development of autonomous robotics in agriculture", Nature Food, Vol. 2 No. 5, pp. 306-309.

Ross, A., Brunius, C., Chevallier, O., Dervilly, G., Elliott, C., Guitton, Y., Prenni, J.E., Savolainen, O., Hemeryck, L., Vidkjær, N.H., Scollan, N., Stead, S.L., Zhang, R. and Vanhaecke, L. (2020), "Making complex measurements of meat composition fast: application of rapid evaporative ionisation mass spectrometry to measuring meat quality and fraud", Meat Science, Vol. 181, p. 108333.

Shahzada, K., Yurkewich, A., Xu, R. and Patel, R.V. (2016), "Sensorization of a surgical robotic instrument for force sensing", SPIE Proc. Optical Fibers and Sensors for Medical Diagnostics and Treatment Applications XVI, 97020U, pp. $1-10$.

Slanger, W.D. and Marchello, M.J. (1994), "Bioelectrical impedance can predict skeletal muscle and fat-free skeletal muscle of beef cow primal cuts", fournal of Animal Science, Vol. 72 No. 12, pp. 3124-3130. 
Swain, B., Halder, J., Sahany, S., Nayak, P.P. and Bhuyan, S. (2021), "Artificial intelligence based human-assisted multipurpose robot", Current Advances in Mechanical Engineering, ICRAMERD 2020, Singapore, Springer, pp. 645-655.

Tae Mun, P., Seung Yeon, W., Sang Ryong, L. and Sziebig, G. (2016), "Force feedback based gripper control on a robotic arm", IEEE 20th fubilee International Conference on Intelligent Engineering Systems (INES), Piscataway, NF, IEEE, pp. 107-112.

Teixeira, A., Silva, S. and Rodrigues, S. (2019), "Chapter six advances in sheep and goat meat products research", Advances in Food and Nutrition Research, Vol. 87, pp. 305-370.

Thampi, A., Hitchman, S., Coen, S. and Vanholsbeeck, F. (2019), "Optical coherence tomography to predict the quality of meat", SPIE Proc. Optical Coherence Imaging Techniques and Imaging in Scattering Media III, p. 110781T.

Thampi, A., Hitchman, S., Coen, S. and Vanholsbeeck, F. (2020), "Towards real time assessment of intramuscular fat content in meat using optical fiber-based optical coherence tomography", Meat Science, Vol. 181, p. 108411.

Usamentiaga, R., Venegas, P., Guerediaga, J., Vega, L., Molleda, J. and Bulnes, F.G. (2014), "Infrared thermography for temperature measurement and nondestructive testing", Sensors, Vol. 14 No. 7, pp. 12305-12348.

Wang, R. (2018), "Biofilms and meat safety: a mini-review", fournal of Food Protection, Vol. 82 No. 1, pp. 120-127.

Xiong, Z., Sun, D.-W., Pu, H., Gao, W. and Dai, Q. (2017), "Applications of emerging imaging techniques for meat quality and safety detection and evaluation: a review", Critical Reviews in Food Science and Nutrition, Vol. 57 No. 4, pp. 755-768.

Zhenjie, F., Qiang, H., Yonglei, Z., Wei, R., Jun-Yi, G., Tao, L., Cheng, C., Wencong, L., Shixun, C., Jincang, Z. and Tongyi, Z. (2019), "Method of artificial intelligence algorithm to improve the automation level of Rietveld refinement", Computational Materials Science, Vol. 156, pp. 310-314.

Zoran, A., Gong, N.-W., Shilkrot, R., Yan, S. and Maes, P. (2015), "Cutting edge vision: metal embedded optics for smart knives", Proceedings of the 33rd Annual ACM Conference Extended Abstracts on Human Factors in Computing Systems, Seoul, Republic of Korea, pp. 1-6.

\section{Corresponding author}

Alex Mason can be contacted at: alex.mason@nmbu.no 